\title{
Evaluation of Hemostasis in Female Dogs with Mammary Carcinoma and the Influence of Clinical Stage and Histopathological Grade
}

\author{
Jéssica Rodrigues de Oliveira, Annelise Carla Camplesi, Sabryna Gouveia Calazans, \\ Aureo Evangelista Santana, Priscila Silva, Bruna Fernanda Firmo, \\ Amanda Bizare \& Márcia Ferreira da Rosa Sobreira
}

\begin{abstract}
Background: Mammary tumors are frequent in female dogs, and are biologically similar in female dogs and humans. Hemostatic disorders are common in humans with cancer, and the mechanisms that conduct coagulation activation involve tissue and cancer procoagulant factors and inflammatory cytokines. Despite the importance of coagulopathy diagnosis that evaluate hemostasis, veterinarians rarely request such tests in routine clinical oncology. This study aimed to investigate the possible hemostatic abnormalities in female dogs with mammary carcinomas and assess any associations with prognostic factors for mammary cancer in female dogs.

Materials, Methods \& Results: A total of 62 female dogs were selected for inclusion in this study regardless of age or breed. The control group consisted of 30 healthy dogs, and the carcinoma group consisted of 32 dogs with a histopathological diagnosis of mammary carcinoma that was free of distant metastases and comorbidities that could interfere with the hemostatic system integrity. The dogs with mammary carcinoma were divided into subgroups according to their histological type, histopathological grading, tumor size, and clinical stage of the disease (TNM) to evaluate the relationships between the subgroups and hemostatic parameters (blood platelet counts, prothrombin time [PT], activated partial thromboplastin time [aPTT], and plasma fibrinogen concentration). The hemostatic parameters were significantly higher in the carcinoma group than in the control group $(P \leq 0.05)$. These findings indicate that there is a strong association between the occurrence of mammary carcinoma and abnormal hemostatic parameters, as well as a strong association between histopathological grade I and normal hemostatic parameters. In addition, when evaluating subgroups, PT and aPTT values were significantly higher in dogs with histopathological grade II and III tumors than in dogs with histopathological grade I tumors. Furthermore, PT and aPTT values were higher in the clinical stage IV subgroup than in the remaining subgroups. Nonetheless, there was no statistical difference in these values among the clinical stage I, III, and IV subgroups.

Discussion: Recent studies demonstrated that thrombocytosis is a common finding in dogs with cancer and that the true incidence of thrombocytosis might be underreported. The prolonged PT and aPTT observed in this study were higher than those described in the literature. The abnormalities in PT and aPTT values detected in female dogs with mammary carcinoma in this study might have been affected by the histopathological severity (grades II and III) and clinical disease stage. Hyperfibrinogenemia detected in the carcinoma group was the most common hemostatic abnormality. This finding was close to that found in the literature, in which $66.7 \%$ of female dogs presented with high fibrinogen values, which was more evident in dogs of clinical disease stages III and IV. The results indicate that $56 \%$ of animals presented with at least three hemostatic abnormalities, including the prolongation of PT and aPTT and hyperfibrinogenemia. These abnormalities were related to advanced clinical stages and histopathological grades II and III. Thus, the observed abnormalities can be indicative of a poorer prognosis and survival time of these patients because the previously identified products of coagulation are important for both tumor dissemination and progression.
\end{abstract}

Keywords: mammary neoplasia, dogs, coagulopathies, platelets, fibrinogen. 


\section{INTRODUCTION}

Hemostatic abnormalities are common in humans [1] and dogs with cancer [5,15] and significantly contribute to the morbidity and mortality rates of this illness [13]. There is a strong association between malignant disease and the occurrence of hemostatic abnormalities, including thrombosis, in humans $[10,13]$.

The pathogenesis of hemostatic disorders associated with malignant neoplasms is complex and multifactorial $[10,12,13]$. The important role of platelets and coagulation and fibrinolysis proteins on tumor growth and progression has been reported [2]. Malignant cells are capable of activating hemostasis [25] and the same oncogenes responsible for neoplastic cell transformation [16] enhance this phenomenon.

Cancer patients typically present with abnormal results on hemostasis evaluation laboratory tests. Previous findings indicated that fibrin formation and degradation was parallel to the development of malignancy, which suggests that fibrin and other coagulation products are important for both tumor thrombogenesis and progression [13]. Human patients with malignant diseases can develop arterial thrombosis and disseminated intravascular coagulation (DIC), followed by severe hemorrhage. Therefore, it is of extreme clinical importance to prevent such complications, because they contribute considerably to the patient's prognosis [12].

The objective of this study was to investigate possible hemostatic abnormalities in female dogs with mammary carcinoma, and assess the associations of such abnormalities with known prognostic factors for female breast cancer.

\section{MATERIALS AND METHODS}

\section{Animals}

A total of 62 female dogs were included in this study, regardless of age or breed. The control group consisted of $30 \mathrm{dogs}$ that were free of mammary neoplasia and presented with clinically healthy laboratory parameters within the reference intervals for the species. Thus, 32 female dogs with a diagnosis of mammary carcinoma determined by histopathological examination, that were confirmed to be free of distant visible metastases by conventional methods of three-dimensional analysis of thoracic radiographs and abdominal ultrasonography, were included as the experimental (carcinoma) group. Female dogs pre- senting with comorbidities or those receiving medications that could interfere with the hemostatic system integrity were excluded. Animals were recruited from the Obstetrics and Oncology Division of Governador Laudo Natel Veterinarian Hospital - FCAV-UNESP and from Veterinarian Hospital Unit of Moura Lacerda University Center, Ribeirão Preto SP Campus. All dogs underwent laboratory tests (blood count, creatinine, and alanine aminotransferase testing). Female dogs with mammary carcinoma were classified according to the clinical stage (I-IV), as proposed by Cassali et al. [4]. In addition, epithelial tumors were classified by specific histopathological criteria based on Elston and Ellis's [9] classification system to determine possible associations with hemostatic parameters (platelet count, PT, aPTT, and plasma fibrinogen concentration).

\section{Blood sampling}

All female dogs with mammary neoplasia were submitted to a mastectomy mammary chain and histopathological examination. Blood samples were collected before the surgical procedure. Animals underwent blood collection by means of jugular venipuncture with the aid of hypodermic needles $(25 \times 0.8$ $\mathrm{mm}$ ) and $5 \mathrm{~mL}$ syringes. The initial $4 \mathrm{~mL}$ of blood was collected in a tube containing sodium chloride citrate anticoagulation at $3.2 \%$ for hemostatic testing, and the remaining $1 \mathrm{~mL}$ of blood was decanted into tubes containing $7.2 \mathrm{mg}$ of ethylenediamine tetra-acetic dipotassium anticoagulant acid ( $\mathrm{K}_{2}$ EDTA) to determine the platelet counts.

Tubes containing blood samples with $\mathrm{K}_{2}$ EDTA were immediately processed and analyzed, whereas tubes containing blood samples with sodium citrate were centrifuged at $1,260 \mathrm{~g}$ for $30 \mathrm{~min}$ at $4^{\circ} \mathrm{C}$. Later, the citrated plasma was split into fractions and stored in labeled micro tubes at $-80^{\circ} \mathrm{C}$ until analysis of the fibrinogen concentration, PT, and aPTT.

\section{Blood sample processing}

Hemostatic tests were performed at the Pathology Laboratory at the "Prof. Joaquim Martins Ferreira Neto" Veterinarian Clinic in Governador Laudo Natel Veterinarian Hospital - FCAV-UNESP, and at the Veterinarian Hospital Unit of Moura Lacerda University Center, Ribeirão Preto Campus, SP. The blood collected in tubes with K2EDTA was homogenized, and the global platelet count was assessed using an automatic blood cell counter (ABC VetCounter) ${ }^{1}$. 
To determine the PT, aPTT, and plasma fibrinogen concentration, the citrate plasma aliquots were thawed to $22 \pm 2^{\circ} \mathrm{C}$, and the analyses were conducted immediately according to the coagulometer manufacturer's guidelines (Quick Timer II)2. The model establishes the coagulation time of a blood plasma sample $(150 \mu \mathrm{L})$ using an optical system to detect sudden optical density variation in the sample, at the coagulation moment. Sets of commercial reagents 3 were used to determine such variations. All samples were double analyzed. The PT and aPTT reference limits were determined from the mean values of the control group and were considered to be altered when the difference was greater than $5 \mathrm{~s}$ of the mean. The platelet and fibrinogen reference limits were determined from local reference values (Laboratory of Veterinarian Clinical Pathology of FCAV/UNESP - Jaboticabal).

\section{Statistical analysis}

The Kolmogorov-Smirnov test for normality was used to evaluate the data normality. A Student's t-test was applied to compare the means of the hemostatic parameters between the control group and the carcinoma group. An analysis of variance was used to compare hemostatic parameters (platelet counts, PT, aPTT, and fibrinogen concentration) among the different subgroups evaluated (histopathological type and grading, clinical disease stage, and tumor size). Tukey's multiple comparison test was used to determine the differences among the mean values of the hemostatic parameters. The analyses were performed using R Software with a significance level $(\alpha)$ of $5 \%$. Multiple correspondence analysis (MCA) using the Chi squared test $\left(\chi^{2}\right)$ was used to evaluate the possible associations among the hemostatic parameters and the different groups and subgroups of female dogs with mammary carcinoma. Values $>1.9$ indicate highly meaningful categories, and values $<1.9$ indicate weakly meaningful categories. MCA statistical analyses were performed using Statistica $7 \circledR$ software

\section{RESULTS}

Significant differences were detected in all hemostatic parameters evaluated in this study (platelet counts, PT, aPTT, and fibrinogen concentration) between the carcinoma and control groups (Table 1). The blood platelet count was significantly higher in the carcinoma group than in the control group. In addition, the blood platelet count was above the reference limit value $(400 \times 103 / \mu \mathrm{L})$ for the species in $46 \%(n=15)$ of the dogs with mammary carcinoma. However, there was no significant difference in the platelet counts among the subgroups (Table 2).

The mean plasma fibrinogen concentration was also significantly higher in the carcinoma group than in the control group (Table 1). Hyperfibrinogenemia (> 300 $\mathrm{mg} / \mathrm{dL})$ was observed in $65.6 \%(\mathrm{n}=21)$ of the sick individuals. In the evaluation of subgroups in Table 2, dogs with histopathological grade II and III presented with significantly higher fibrinogen concentration values than those of dogs with grade I tumors. Regarding clinical staging, dogs with stage IV carcinoma presented with the highest mean fibrinogen value, which was significantly higher than those of dogs with stage I and II carcinoma.

The mean PT and aPTT values were significantly higher in the carcinoma group than in the control group (Table 1). Evaluation of the subgroups (Table 2) revealed that the PT and aPTT values were higher in dogs with histopathological grade II and III tumors than in those with grade I tumors. With regard to the clinical stage, the PT and aPTT values were significantly higher in dogs with stage IV carcinoma than in dogs with stage II carcinoma. The evaluation of tumor size indicated that dogs with tumors of $3-5 \mathrm{~cm}$ in diameter presented with significantly lower aPTT values than did the dogs with other tumor sizes. In the histopathological subgroup analysis, no meaningful differences were observed in the PT and aPTT values (Table 2).

Perceptual mapping indicated two groups that contained both interrelating and contrasting categories (Figure 1), and four groups of interrelating categories (Figure 2).

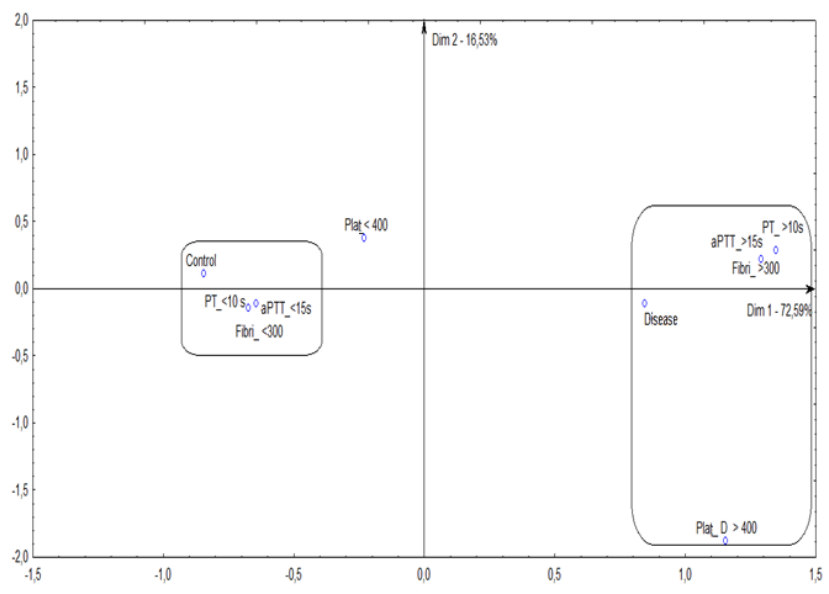

Figure 1. Perceptual mapping of the hemostatic parameters in the control and carcinoma group. The parameters presented in the map are platelets (Plat), prothrombin time (PT), activated partial thromboplastin time (aPTT), and fibrinogen (Fibri). Rectangles represent strong associations. The perceptual mapping quality was $89.12 \%$ (dimension $1: 72.59 \%$ and dimension 2: $16.53 \%$ ). 


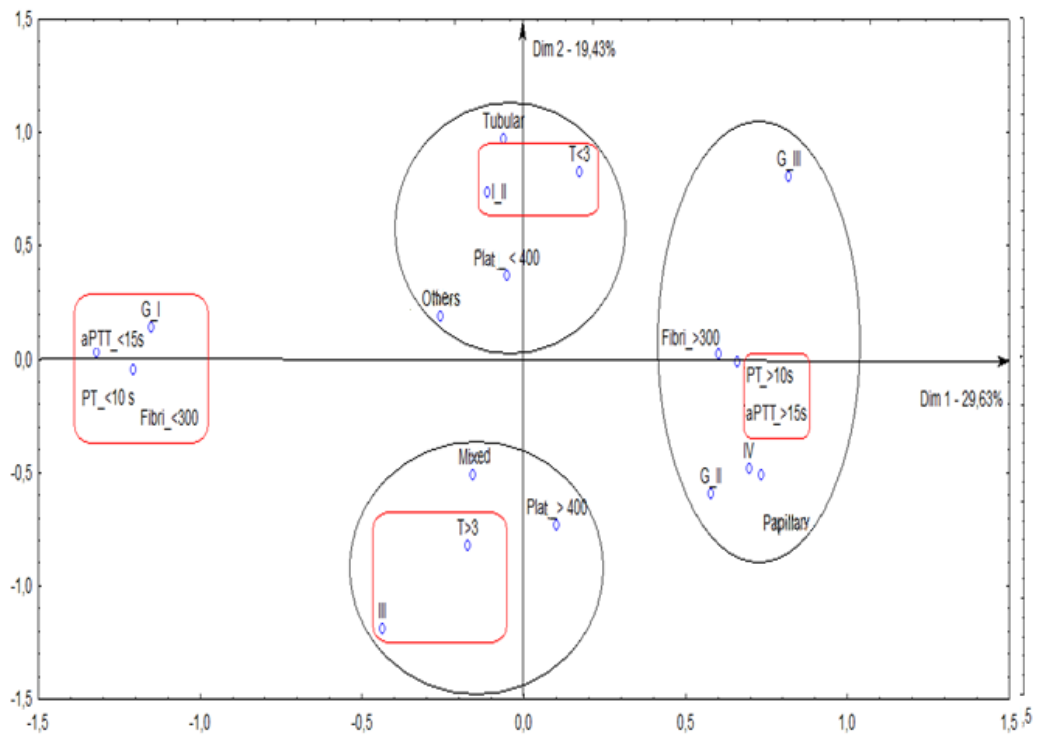

Figure 2. Perceptual mapping of the hemostatic parameters (Plat, PT, aPTT and Fibri) obtained among in the subgroups studied (Histopathological Type mixed, papillary, tubular, others; Clinical Staging I_II, III, IV; Histopathological Degree G_I, G_II, G_III and tumor size T S

3 and $\mathrm{T} \geq 3$ ). Rectangles represent strong associations and circles represent weak associations.

The perceptual mapping quality was $49.06 \%$ (dimension $1: 29.63 \%$ and dimension $19.43 \%$ ).

Table 1. Comparison of the hemostatic parameters (platelets count, prothrombin time, activated partial thromboplastin time and fibrinogen concentration) in the control (healthy) and mammary carcinoma groups (diseased).

\begin{tabular}{ccc}
\multirow{2}{*}{ Parameter } & \multicolumn{2}{c}{ Groups } \\
\cline { 2 - 3 } & $299.5 \pm 99.1^{\mathrm{b}}$ & $417.7 \pm 166.2^{\mathrm{a}}$ \\
\hline Platelets $\left(\mathrm{x} 10^{3}\right) / \mu \mathrm{L}$ & $6.5 \pm 0.9^{\mathrm{b}}$ & $15.2 \pm 7.1^{\mathrm{a}}$ \\
PT (s) & $9.2 \pm 1.6^{\mathrm{b}}$ & $20.2 \pm 10.3^{\mathrm{a}}$ \\
aPTT (s) & $251.4 \pm 32.8^{\mathrm{b}}$ & $385.0 \pm 136.0^{\mathrm{a}}$ \\
\hline Fibrin $(\mathrm{mg} / \mathrm{dL})$ &
\end{tabular}

Mean values of the hemostatic parameters a,bDifferent characters indicate significant differences $(P \leq 0.05)$ between the two groups. $\mathrm{PT}=$ prothrombin time, aPTT $=$ activated partial thromboplastin time.

\section{DISCUSSION}

The conducting mechanisms that activate the hemostatic system in patients with cancer involve tissue and cancer pro-coagulant factors and inflammatory cytokines [25]. However, it is well established that hemostatic compounds such as platelets and coagulation and fibrinolysis proteins play important roles in tumor growth and progression [2].

In this study, the platelet count was higher in the carcinoma group than in the control group. Thrombocytosis is a rare condition in dogs with malignant neoplasia [5], but typically occurs in humans with cancer [6]. However, there ports presented in the literature are conflicting, as some authors suggest that thrombocytopenia is the most common abnormality in dogs with malignant disease [5], while others indicate that thrombocytosis is a frequent abnormality in dogs with cancer [18,21,27]; thus, the true prevalence of thrombocytosis in animals with neoplasia might be underreported [5].

Thrombocytosis is associated with the state of hypercoagulability that follows cancer and with poor prognosis in human patients $[14,24]$. The mechanism underlying thrombocytosis in patients with neoplasia has not been fully elucidated, but it has been suggested that this disorder is due to the release of proangiogenic tumor factors and inflammatory cytokines (interleukin [IL]-1 and [IL]-6) or the hematopoietic growth factors $[5,21]$ expelled or induced by the tumor. 
Table 2. Platelet count, prothrombin time, activated partial thromboplastin time, and fibrinogen concentration among the subgroups of the female dogs with mammary carcinoma (histopathological grade, clinical staging and tumor size).

\begin{tabular}{|c|c|c|c|c|}
\hline Carcinoma & $\begin{array}{l}\text { Platelets } \\
\left(\mathrm{x} 10^{3}\right) / \mu \mathrm{L}\end{array}$ & $\mathrm{PT}(\mathrm{s})$ & aPTT (s) & Fibrin $(\mathrm{mg} / \mathrm{dL})$ \\
\hline \multicolumn{5}{|l|}{ Histopathological Type } \\
\hline Adenomyoepithelioma Malignant & $375.5 \pm 38.9^{\mathrm{a}}$ & $11.6 \pm 10.7^{\mathrm{a}}$ & $16.6 \pm 15.3^{\mathrm{a}}$ & $396 \pm 148.5^{\mathrm{a}}$ \\
\hline Mixed tumor carcinoma & $421.6 \pm 130.8^{a}$ & $14.5 \pm 6.6^{\mathrm{a}}$ & $19.2 \pm 8.4^{\mathrm{a}}$ & $353.4 \pm 125.3^{\mathrm{a}}$ \\
\hline Papillary carcinoma & $458.2 \pm 226.6^{\mathrm{a}}$ & $19.4 \pm 7.5^{\mathrm{a}}$ & $25.9 \pm 9.7^{\mathrm{a}}$ & $481.2 \pm 124.7^{\mathrm{a}}$ \\
\hline Solid carcinoma & $339.7 \pm 170.2^{\mathrm{a}}$ & $20.3 \pm 10.8^{\mathrm{a}}$ & $29.2 \pm 15.9^{\mathrm{a}}$ & $523.3 \pm 196.1^{\mathrm{a}}$ \\
\hline Tubular carcinoma & $425.1 \pm 219.8^{\mathrm{a}}$ & $12.7 \pm 5.3^{\mathrm{a}}$ & $16.1 \pm 9.6^{\mathrm{a}}$ & $325.6 \pm 94.5^{\mathrm{a}}$ \\
\hline \multicolumn{5}{|l|}{ Histopathological Grading } \\
\hline Grade 1 & $424.8 \pm 164.6^{\mathrm{a}}$ & $9.2 \pm 4.4^{\mathrm{b}}$ & $12.2 \pm 6.2^{\mathrm{b}}$ & $292.0 \pm 86.1^{b}$ \\
\hline Grade 2 & $444.6 \pm 132.7^{\mathrm{a}}$ & $18.9 \pm 5.9^{\mathrm{a}}$ & $23.4 \pm 7.8^{\mathrm{a}}$ & $427.6 \pm 129.2^{\mathrm{a}}$ \\
\hline Grade 3 & $358.3 \pm 224.9^{\mathrm{a}}$ & $19.9 \pm 5.4^{\mathrm{a}}$ & $29.7 \pm 9.4^{\mathrm{a}}$ & $484.7 \pm 127.4^{\mathrm{a}}$ \\
\hline \multicolumn{5}{|l|}{ Clinical Staging } \\
\hline Stage I & $382.9 \pm 158.8^{\mathrm{a}}$ & $14.3 \pm 5^{\mathrm{a}, \mathrm{b}}$ & $18.4 \pm 6.8^{\mathrm{a}, \mathrm{b}}$ & $338.1 \pm 88.1^{\mathrm{b}}$ \\
\hline Stage II & $502.8 \pm 246.4^{\mathrm{a}}$ & $8.1 \pm 5.4^{b}$ & $8.5 \pm 4.4^{\mathrm{b}}$ & $301.3 \pm 58.4^{\mathrm{b}}$ \\
\hline Stage III & $449.9 \pm 170.1^{\mathrm{a}}$ & $15.2 \pm 8.6^{\mathrm{a}, \mathrm{b}}$ & $20.6 \pm 10.9^{\mathrm{a}, \mathrm{b}}$ & $372.4 \pm 165^{\mathrm{a}, \mathrm{b}}$ \\
\hline StageIV & $406.4 \pm 140.7^{\mathrm{a}}$ & $21.1 \pm 6.6^{\mathrm{a}}$ & $30.3 \pm 10^{\mathrm{a}}$ & $539.3 \pm 110^{\mathrm{a}}$ \\
\hline \multicolumn{5}{|l|}{ Tumor size } \\
\hline Size $<3 \mathrm{~cm}$ & $382 \pm 158.1^{\mathrm{a}}$ & $15.3 \pm 5.8^{\mathrm{a}}$ & $21.1 \pm 9.8^{\mathrm{a}}$ & $367.8 \pm 117.9^{a}$ \\
\hline Size $3-5 \mathrm{~cm}$ & $502.8 \pm 246.4^{\mathrm{a}}$ & $8.1 \pm 5.4^{\mathrm{a}}$ & $8.5 \pm 4.4^{b}$ & $301.3 \pm 58.4^{\mathrm{a}}$ \\
\hline Size $>5 \mathrm{~cm}$ & $436.8 \pm 149.3^{\mathrm{a}}$ & $17.3 \pm 8.2^{\mathrm{a}}$ & $23 \pm 10^{\mathrm{a}}$ & $435.8 \pm 162.7^{a}$ \\
\hline
\end{tabular}

Mean values of the hemostatic parameters in the subgroups. ${ }^{\text {a,b }}$ Different characters indicate significant differences $(P \leq 0.05)$ between the evaluated groups. $\mathrm{PT}=$ prothrombin time, $\mathrm{aPTT}=$ activated partial thromboplastin time.

Prolongation of PT and aPTT was observed in $62.5 \%$ of the dogs in the carcinoma group, and this result is superior to that reported by Stockhaus et al. [22]. This study demonstrated a strong association between the presence of carcinoma and the evaluated hemostatic parameters (Figure 1), and found significant differences between the two groups (Table 1). The current results are in contrast to those of the study conducted by Saavedra et al. [21], where in no meaningful differences in PT and aPTT were observed between dogs with carcinoma and the control group.

The hemostatic abnormalities identified in female dogs with mammary carcinoma in this study might have been affected by the histopathological grade and clinical stage of the disease. Therefore, these characteristics are associated with more aggressive tumor malignancy, and likely to greater induction of inflammatory cytokines, which can promote increases in the levels of coagulation factors [1]. However, the exact causes of coagulation abnormalities remain unknown. Thus, the present result suggests that the actual incidence of hemostatic abnormalities in animals with neoplasia might be underestimated.

The present study indicates that female dogs in clinical stages I, III, and IV exhibited higher PT and aPTT values. The abnormalities observed in dogs at stages III and IV corroborate the findings of the study by Stockhaus et al. [22], in which prolongation of PT and aPTT was also observed in dogs at the same disease stages. However, such abnormalities were not expected at stage I. The fact that such animals present with moderately differentiated (histopathological grade II) and slightly differentiated (histopathologicalgrade III) tumors might explain the results. These grades indicate greater tumor aggression and malignancy, thereby justifying the abnormalities observed in the hemostatic parameters (aPTT, PT, and fibrinogen concentration) [Table 2]. A strong association was observed between 
histopathological grade I and hemostatic parameters (aPTT, PT, and fibrinogen concentration) within the normal range (Figure 2), indicating that the female dogs with mammary carcinoma grade I did not present with coagulation abnormalities and were similar to the dogs in the control group.

Hyperfibrinogenemia is common in dogs with malignant disease [26], and was the most frequent hemostatic abnormality found in this study; $65 \%$ of female dogs with mammary carcinoma presented with hyperfibrinogenemia. The prevalence increased according to the progression of the illness, and was more evident in dogs at disease stages III and IV, as well as in dogs with highly aggressive tumors of histopathological grades II and III. This result is similar to that previously reported [7], in which $66.7 \%$ of the female dogs presented with high fibrinogen values that became more evident in dogs at stages III and IV.

Despite the increasing levels of fibrinogen with disease progression in female dogs with mammary carcinoma, the correlation between the fibrinogen concentration and tumor progression remains controversial and unclear [19,21,22]. However, hyperfibrinogenemia has previously been associated with cancer progression in human patients [20] and canine patients [1]. In addition, $61 \%$ of dogs with carcinoma and hypercoagulability were found to present with increased fibrinogen levels, thus indicating the effect of the fibrinogen on hypercoagulability [21].

DIC was reported in $\leq 10 \%$ of dogs with solid tumors [21] and has been identified in female dogs with mammary carcinoma [17]. DIC diagnosis is based on the presence of at least three abnormalities including thrombocytopenia; prolongation of PT, aPTT, and thrombin time; hyperfibrinogenemia; and increases in the products of fibrin degradation and D-dimerization $[3,22,23]$. However, none of the dogs included in this study were diagnosed with DIC. Nonetheless, it is important to mention that $56 \%$ of the animals in this study presented with at least three coagulation abnormalities including PT and aPTT prolongation and hyperfibrinogenemia. This indicates that female dogs with mammary carcinoma may present with blood coagulation abnormalities without apparent clinical manifestations of thrombosis or hemorrhage. Thus, the current study demonstrates the importance of the evaluation of hemostasis in female dogs with mammary carcinoma.

\section{CONCLUSIONS}

In conclusion, the majority of female dogs with mammary carcinoma presented with abnormalities on blood coagulation laboratory tests, and such abnormalities were influenced by the histopathological grade and clinical disease stage. Hyperfibrinogenemia was the most common abnormality in female dogs with mammary carcinoma and seems to be related to disease progression. The increases in the platelet count, fibrinogen concentration, PT, and aPTT might be associated with a pro-inflammatory response induced by the tumor and are indicative of a state of hyperfibrinogenemia.

\section{MANUFACTURERS}

${ }^{1}$ Horiba Medical. Montpellier, France.

${ }^{2}$ Drake Eletrônica e Comércio Ltda. São Jose do Rio Preto, SP, Brazil. ${ }^{3}$ Labtest - Sistema de Diagnósticos Ltda. Lagoa Santa, MG, Brazil.

Ethical approval. The experimental protocol of this study is in accordance with the ethical principles for animal experimentation adopted by COBEA (BrazilianAnimalExperimental Council) and approved by ethics committee of FCAV-UNESP, protocol number 009735-14.

Declaration of interest. The authors report no conflicts of interest. The authors alone are responsible for the content and writing of the paper.

\section{REFERENCES}

1 Andreasen E.B., Tranholm M., Wiinberg B., Markussen B. \& Kristensen A.T. 2012. Hemostatic alterations in a group of canine cancer patients are associated with cancer type and disease progression. Acta Veterinaria Scandinavica. 54(3): 1-8.

2 Bogenrieder T. \& Herlyn M. 2003. Axis of evil: molecular mechanisms of cancer metastasis. Oncogene. 22(42): 6524-6536.

3 Brooks M.B. \& Laforcade A. 2010. Acquired coagulopathies. In: Schalm's Veterinary Hematology. Ames: Blackwell Publishing, pp.654-660. 
4 Cassali G.D., Lavalle G.E., Ferreira E., Estrela-lima A., De nardi A.B., Ghever C., Sobral R.A., Amorim R.L., Oliveira L.O., Sueiro F.A.R., Beserra H.E.O., Bertagnolli A.C., Gamba C.O., Damasceno K.A., Campos C.B., Araujo M.R., Campos L.C., Monteiro L.N., Nunes F.C., Horta R.S., Reis D.C., Luvizott M.C.R., Magalhães G.M., Raposo J.B., Ferreira A.M.R., Tanaka N.M., Grandi F., Ubukata R., Batschinski K., Terra E.M., Salvador R.C.L., Jark P.C., Delecrodi J.E.R., Nascimento N.A., Silva D.N., Silva L.P., Ferreira K.C.R.S., Frehse M.S., Di santis G.W., Silva E.O., Guim T.N., Kerr B., Cintra P.P., Silva F.B.F., Leite J.S., Mello M.F.V., Ferreira M.L.G., Fukumasu H., Salgado B.S. \& Torres R. 2014. Consensus for the Diagnosis, Prognosis and Treatment of Canine Mammary Tumors. Brazilian Journal of Veterinary Pathology. 7(2): 38-69.

5 Childress O.M. 2012. Hematologic Abnormalities in the Small Animal Cancer Patient. Veterinary Clinics of North America: Small Animal Practice. 42(1): 123-155.

6 Desancho M.T. \& Rand J.H. 2010. Coagulopathic complications of cancer patients. In: Cancer medicine. In: Hong W.K., Bast R.C., Hait W.N., Kufe D.W., Pollock R.E., Weichselbaum R.R., Holland J.F. \& Frei III E. (Eds). 8th edn. Selton: PMPH-USA, pp.1813-1822.

7 Duda N.C.B., Valle S.F., Matheus J.P., Angeli N.C., Vieira L.O., Sonne L. \& González F.H.D. 2017. Paraneoplastic hematological, biochemical, and hemostatic abnormalities in female dogs with mammary neoplasms. Pesquisa Veterinária Brasileira. 37(5): 479-484.

8 Edwards R.L., Rickles F.R., Moritz T.E., Henderson W.G., Zacharski L.R., Forman W.B., Cornelli C.J., Forcier R.J., O'donnell J.F., Headley E., Kim S.H., O'dell R., Tornyos K. \& Kwaan H.C. 1987. Abnormalities of blood coagulation tests in patients with cancer. American Journal of Clinical Pathology. 88(5): 596-602.

9 Elston C.W. \& Ellis I.O. 1998. Assessment of histological grade. In: Elston C.W. \& Ellis I.O. (Eds). Systemic pathology - The breast. London: Churchill \& Livingstone, pp.365-384.

10 Falanga A., Marchetti M. \& Russo L. 2015. The mechanisms of cancer-associated thrombosis. Thrombosis Research. 135(Suppl 1): S8-S11.

11 Falanga A., Marchetti M. \& Vignoli A. 2013. Coagulation and cancer: biological and clinical aspects. Journal of Thrombosis and Haemostasis. 11(2): 223-233.

12 Falanga A., Russo L. \& Milesi V. 2014. The coagulopathy of cancer. Current Opinion in Hematology. 21(5): 423-429.

13 Falanga A., Russo L., Milesi M. \& Vignoli A. 2017. Mechanisms and risk factors of thrombosis in cancer. Critical Reviews in Oncology/Hematology. (118): 79-83.

14 Gungor T., Kanat-Pektas M., Sucak A. \& Mollamahmutoglu L. 2009. The role of thrombocytosis in prognostic evaluation of epithelial ovarian tumors. Archives of Gynecology and Obstetrics. 279(1): 53-56.

15 Kristensen A.T., Wiinberg B., Jessen L.R., Andreasen E. \& Jensen A.L. 2008. Evaluation of human recombinant tissue factor activated thromboelastography in $49 \mathrm{dogs}$ with neoplasia. Journal of Veterinary Internal Medicine. 22(1): 140-147.

16 Magnus N., D’Asti E., Meehan B., Garnier D. \& Rak J. 2014. Oncogenes and the coagulation system-forces that modulate dormant and aggressive states in cancer. Thrombosis Research. 133(Suppl 2): S1-S9.

17 Mischke R., Wohlsein P., Busse L. \& Pohlenz J. 1998. Disseminated intravascular coagulation and hyperfibrinolysis in dogs with metastasizing mammary carcinoma. Schweizer Archivfür Tierheilkunde. 140(12): 497-505.

18 Neel J.A., Snyder L. \& Grindem C.B. 2012. Thrombocytosis: a retrospective study of 165 dogs. Veterinary Clinical Pathology. 41(2): 216-222.

19 O'Donnell M.R., Slichter S.J., Weiden P.L. \& Storb R. 1981. Platelet and fibrinogen kinetics in canine tumors. Cancer Research. 41(4): 1379-1383.

20 Rickles F.R., Levine M. \& Edwards R.L. 1992. Hemostatic alterations in cancer patients. Cancer and Metastasis Reviews. 11(3-4): 237-248.

21 Saavedra P.V., García A.L., López S.Z. \& Couto G. 2011. Hemostatic abnormalities in dogs with carcinoma: A thromboelastographic characterization of hypercoagulability. The Veterinary Journal. 190(2): e78-e83.

22 Stockhaus C., Kohn B., Rudolph R., Brunnberg L. \& Giger U. 1999. Correlation of haemostatic abnormalities with tumor stage and characteristics in dogs with mammary carcinoma. The Journal of Small Animal Practice. 40(7): 326-331.

23 Stokol T., Brooks M., Erb H. \& Mauldin G.E. 1999. Evaluation of kits for the detection of fibrin(ogen) degradation products in dogs. Journal of Veterinary Internal Medicine. 13(5): 478-484. 
J.R. Oliveira, A.C. Camplesi, S.G. Calazans, et al. 2019. Evaluation of Hemostasis in Female Dogs with Mammary Carcinoma and the Influence of Clinical Stage and Histopathological Grade. Acta Scientiae Veterinariae. 47: 1705.

24 Symbas N.P., Townsend M.F., El-Galley R., Keane T.E., Graham S.D. \& Petros J.A. 2000. Poor prognosis associated with thrombocytosis in patients with renal cell carcinoma. BJU International. 86(3): 203-207.

25 Tabak D., Torres L.G. \& Nahoum B. 2011. Câncer e trombose. Onco \&. 4: 26-32.

26 Wada H., Sase T. \& Yamaguchi M. 2005. Hypercoagulant states in malignant lymphoma. Experimental Oncology. 27(3): 179-185.

27 Woolcock A.D., Keenan A., Cheung C., Christian J.A. \& Moore G.E. 2017. Thrombocytosis in 715 Dogs (20112015). Journal of Veterinary Internal Medicine. 31(6): 1691-1699. 